\title{
O JORNALISMO IMPRESSO NA COMUNIDADE UNIVERSITÁRIA
}

\section{Adriana Strieder Philippsen}

adristrieder@yahoo.com.br

Faculdade Estadual de Educação, Ciências e Letras de Paranavaí

Danielle Peralta

Universidade Estadual de Maringá

dperalta2@uem.br

Sebastião Gazola

Universidade Estadual de Maringá

sgazola@uem.br

Fernanda Lang Schumacher

Universidade Estadual de Maringá

fernandalschumacher@gmail.com

Jennifer Kung

Universidade Estadual de Maringá jenniferkung109@hotmail.com

\section{Resumo}

Este artigo teve com objetivo conhecer a opinião da comunidade acadêmica sobre o Informativo UEM por meio de uma pesquisa quantitativa junto a comunidade universitária da Universidade Estadual de Maringá. Para a obtenção das informações o instrumento utilizado foi um questionário e o tipo de entrevista foi direta. $O$ tipo de levantamento foi por amostragem estratificada proporcional. O levantamento de dados foi realizado no período de agosto a outubro de 2011. O tipo de levantamento foi por amostragem estratificada proporcional. Para as análises utilizou-se a estatística descritiva e investigou-se a associação entre variáveis por meio de testes estatísticos não paramétricos. Os resultados mostraram que a maioria da comunidade universitária lê o Informativo, sendo o meio impresso o mais utilizado. O principal motivo de interesse em ler o Informativo UEM é a busca por informações/soluções para suas necessidades. Pode-se concluir que a maioria dos docentes e agentes considerou tanto o conteúdo quanto o visual do Informativo impresso como bom.

Palavras-chave: Informativo UEM. Pesquisa de opinião. Testes Não-paramétricos. 


\title{
PRINTED IN THE COMMUNITY UNIVERSITY JOURNALISM
}

\begin{abstract}
This article has aimed to know the opinion of the academic community about the Informative UEM through a quantitative survey among the university community of the Universidade Estadual de Maringá. To obtain the information the instrument used was a questionnaire and the type of interview was straightforward. The type of survey was stratified proportional sampling. The survey was conducted from August to October 2011. The type of survey was stratified proportional sampling. For the analyzes used descriptive statistics and investigated the association between variables using non-parametric statistical tests. The results showed that most of the academic community read the informative, the print medium being the most used. The main reason for interest in reading the Informative UEM is the search for information / solutions to your needs. It can be concluded that the majority of faculty and staff considered both the content and the look of the printed newsletter as well.
\end{abstract}

Keywords: Informative UEM. Research opinion. Non-parametric tests. 


\section{INTRODUÇÃO}

O jornal impresso é um instrumento que, ainda nos dias atuais, constitui um veículo de conhecimento que serve para informar e fornecer notícias a uma sociedade. Com o surgimento da Internet e do jornalismo digital, o número de jornais impressos sofreu uma grande queda ano após ano e a quantidade de leitores vem acompanhando essa tendência. Há quem defenda a ideia de que o jornal impresso deixará de circular em breve e, devido a esse fato, o jornal impresso precisou readaptar-se, aprofundar-se na problematização das informações e inovar suas ferramentas para atrair o leitor.

Segundo Santaella (2000) um dos motivos da crise por que passa o jornalismo impresso é o erro que comete de não aprofundar (salvo raras exceções) os fatos dos conteúdos já veiculados pelas rádios e TVs, repetindo o que o leitor já sabia de antemão. Ainda, as mídias eletrônicas e a rede mundial de computadores são os inimigos do jornalismo impresso. Também a publicidade, que é a principal fonte de renda da imprensa impressa foi reduzida drasticamente. Mesmo assim, o jornal impresso, por sua tradição, possui potencial para sobreviver.

A crise do jornal impresso não é atual e vem ocorrendo a décadas de antes do surgimento da internet, porém, com a ascensão da mídia on-line houve um agravamento na crise do jornal impresso (RIGHETTI E CARVALHO, 2008).

No Brasil não se observa uma grande crise no jornal impresso apesar de que o rádio e a TV são de preferência nacional como fonte de notícias. No entanto a internet com quase 40 milhões de usuários é a forma mais preferida de se manter informado configurando uma ameaça ao jornal impresso ${ }^{1}$.

Pesquisa encomendada pela Secretaria de Comunicação (Secom) da Presidência da República aponta que os jornais impressos são lidos por 46,1\% dos brasileiros, enquanto as revistas são consumidas por 34,9\% da população. Os meios de comunicação mais usados são a televisão (96,6\% assistem TV) e o rádio (80,3\% utilizam o rádio). O jornal impresso tem a terceira posição e a internet vem crescendo. De acordo com a pesquisa, a TV aberta é apontada por $69,4 \%$ dos entrevistados como o meio de comunicação mais confiável, seguida

\footnotetext{
${ }^{1}$ http://www.observatoriodaimprensa.com.br/news/view/problemas_e_perspectivas_do_jornal_impresso 
pelo rádio $(7,2 \%)$, internet $(6,5 \%)$ e jornal impresso $(6,3 \%)^{2}$.

O processo de comunicação dentro de Instituições Públicas deve ter como objetivo criar, fortalecer e capacitar os indivíduos para fiscalizarem as ações públicas. A idéia de comunicação comunitária se refere justamente ao uso dos meios de comunicação pelas comunidades. É realizada por meio da participação e do compromisso com a comunidade e tem como característica identificar e transmitir os interesses da comunidade em que está inserida.

A Universidade Estadual de Maringá tem sua sede situada na cidade de Maringá/PR e mantém atividades de ensino, pesquisa e extensão em várias cidades do Estado do Paraná. Sua comunidade universitária é formada por mais de 20 mil pessoas, entre discentes, docentes e técnicos administrativos. A Universidade possui também um Hospital Universitário (HU), localizado na cidade sede.

Segundo Parizotto (2010), o Informativo UEM “é o veículo de comunicação interna da UEM que surgiu para suprir carências na comunicação interna da mesma e se pauta pela democratização das informações que dizem respeito a todos os segmentos da comunidade universitária".

Todos os dias as pessoas buscam notícias e informações. Ao contabilizar as buscas é possível saber quais são os assuntos mais relevantes e os temas que despertam a curiosidade da comunidade acadêmica. Estas buscas se dão através dos diversos recursos tecnológicos e diante dessa realidade, pergunta-se: qual a utilidade para o leitor, do mais tradicional veículo de comunicação, o jornal impresso? (BARREIROS \& PAULA, 2007).

São essas as indagações que este estudo buscou a partir de uma pesquisa quantitativa junto a comunidade universitária da Universidade Estadual de Maringá. Assim, realizou-se uma pesquisa de opinião junto à comunidade universitária da Universidade Estadual de Maringá, com o objetivo de conhecer sua opinião sobre o Informativo UEM.

\section{MATERIAL E MÉTODOS}

Como a maior parte da comunidade acadêmica está situada na sede da Universidade, a pesquisa abrangeu os docentes e agentes universitários da sede e do Hospital Universitário (HU), não incluindo os campi situados em outras cidades. Para o cálculo do tamanho da amostra foi utilizado um nível de confiança de $95 \%$ com erro máximo da estimativa de 5\%.

\footnotetext{
${ }^{2}$ http://www.estadao.com.br/noticias/impresso,pesquisa-mostra-que-461-da-populacao-le-jornal,568419,0.htm 
Para a categoria "Docentes" foi utilizada a amostragem estratificada proporcional por Centros de Ensino e para a categoria "Agentes Universitários" foi utilizado a amostragem estratificada, proporcional por setores. O tamanho da amostra pesquisada foi de 311 docentes e 366 agentes universitários. O instrumento utilizado foi um questionário e o levantamento de dados foi realizado por entrevista direta.

O banco de dados provenientes da coleta de dados foi construído utilizando o software EXCEL e as análises descritivas foram realizadas por meio dos softwares EXCEL e R. Ainda, investigou-se a associação entre variáveis por meio de testes estatísticos não paramétricos.

$\mathrm{Na}$ literatura um dos testes não paramétricos mais utilizado para verificar se existe associação entre duas variáveis qualitativas é o teste qui-quadrado de independência. Com base em uma amostra de observações dispõe-se os dados em uma tabela de contingência e sob a hipótese estatística de que não há associação entre as variáveis, o teste é realizado usando a estatística:

$$
\chi^{2}=\sum_{\mathrm{i}=1}^{\mathrm{L}} \sum_{\mathrm{j}=1}^{\mathrm{C}} \frac{\left(\mathrm{O}_{\mathrm{ij}}-\mathrm{E}_{\mathrm{ij}}\right)^{2}}{\mathrm{E}_{\mathrm{ij}}} \sim \chi_{(\mathrm{L}-1)(\mathrm{C}-1)}^{2}
$$

em que L é o número de linhas e C é o número de colunas da tabela de contingência; Oij é a frequência observada na célula $(i, j)$ da tabela de contingência; Eij é a frequência esperada na célula (i,j), sendo calculada por

$$
E_{i j}=\frac{\text { (total da linha i) } x \text { (total da coluna } j)}{\text { total geral }}, i=1, \ldots, L, j=1, \ldots, C
$$

Segundo Campos (1983), o teste de não é apropriado para casos de pequenas amostras ou quando existirem frequências esperadas inferiores a 5. Em situações como estas recomenda-se a aplicação do Teste Exato de Fisher, que consiste em determinar a exata probabilidade de ocorrência de uma frequência observada ou de valores ainda mais extremos. O método do teste exato de Fisher é: em uma tabela de contingência, com os totais marginais fixos, as frequências observadas têm distribuição hipergeométrica. A exata probabilidade de ocorrência de um valor igual ou mais extremo do que d, é dada por:

$$
p=\sum_{i=0}^{d} p_{i}, \quad \text { onde } \quad p_{i}=\frac{C_{N_{2}}^{i} \cdot C_{N}^{b+d-i}}{C_{N}^{b+d}},
$$

em que a, b, c, d são as frequências observadas, d é a menor das frequências, N1=a+b, N2= $\mathrm{c}+\mathrm{d}$ e $\mathrm{N}$ é o total geral. 


\section{RESULTADOS E ANÁLISE}

Do total de entrevistados, $81 \%$ dos docentes e $73 \%$ dos agentes universitários leem o Informativo UEM. Quanto ao acesso ao Informativo, 97\% dos docentes e 92\% dos agentes universitários, dizem ter acesso fácil ao Informativo. Observou-se também que $52 \%$ dos docentes e $69 \%$ dos agentes acham que o número de exemplares pode ser diminuído. A grande maioria das duas categorias (94\% de docentes e $91 \%$ de agentes) disse que os assuntos abordados pelo Informativo impresso são do seu interesse.

Observou-se que tanto para os docentes quanto para os técnicos a maioria, 77,9\% e $77,2 \%$ respectivamente, utiliza o meio impresso para leitura, enquanto 16,6\% e 13,3\%, respectivamente, leem o Informativo em ambas as versões, on-line e impresso (Tabela 1). $\mathrm{O}$ teste qui-quadrado mostrou não haver associação entre o meio utilizado e a categoria (pvalor $=0,162)$.

\begin{tabular}{lcc}
\hline \multicolumn{1}{c}{ Meio } & Docentes & Agentes \\
\hline Impresso & $77,9 \%$ & $77,2 \%$ \\
On-line & $5,5 \%$ & $9,5 \%$ \\
Impresso \& On-line & $16,6 \%$ & $13,3 \%$ \\
\hline
\end{tabular}

Tabela 1: Preferência quanto ao meio de leitura utilizado por categoria. Fonte: Elaborado pelo autor

A comunidade universitária não tem interesse em obter suas informações utilizando apenas o meio on-line, a Tabela 2 mostra que a maioria dos agentes $(73,5 \%)$ não concordam que o Informativo deve deixar de circular na forma impressa, também com relação aos docentes $(61,9 \%)$ compartilham da mesma opinião. O teste qui-quadrado forneceu pvalor=0,008, indicando que existe associação entre a variável categoria e o informativo deixar de circular em papel.

\begin{tabular}{|c|c|c|}
\hline Opinião & Docentes & Agentes \\
\hline Sim & $38,1 \%$ & $26,5 \%$ \\
\hline Não & $61,9 \%$ & $73,5 \%$ \\
\hline
\end{tabular}

Tabela 2: Opinião do leitor sobre o Informativo deixar de circular em papel e manter apenas no formato digital. Fonte: Elaborado pelo autor

Dos entrevistados que responderam que utilizam os meios impresso e impresso e online (Tabela 3), 52,3\% dos docentes não querem que o Informativo deixe de circular em papel e $65,5 \%$ dos agentes tem a mesma opinião. 


\begin{tabular}{l|cc|cc}
\hline \multirow{2}{*}{ Meio } & \multicolumn{2}{|c|}{ Docentes } & \multicolumn{2}{c}{ Agentes } \\
\cline { 2 - 5 } & Sim & Não & Sim & Não \\
\hline Impresso & $30,1 \%$ & $52,3 \%$ & $20,2 \%$ & $65,1 \%$ \\
Impresso \& On-line & $7,9 \%$ & $9,6 \%$ & $6,3 \%$ & $8,4 \%$ \\
\hline
\end{tabular}

Tabela 3: Meio utilizado para leitura versus deixar de circular em papel para as duas categorias. Fonte: Elaborado pelo autor

Para os que leem o Informativo, docentes e agentes, $43,1 \%$ e 49,8\% respectivamente, o fazem semanalmente. Observa-se que 56,9\% dos docentes e 50,2\% dos agentes leem algumas edições por mês ou eventualmente (Tabela 4).

\begin{tabular}{lcc}
\hline Periodicidade & Docentes & Agentes \\
\hline Semanalmente & $43,1 \%$ & $49,8 \%$ \\
Algumas edições por mês & $31,8 \%$ & $28,1 \%$ \\
Eventualmente & $25,1 \%$ & $22,1 \%$ \\
\hline
\end{tabular}

Tabela 4: Periodicidade com que leem o Informativo. Fonte: Elaborado pelo autor

A maioria da comunidade universitária $(55,2 \%)$ dos docentes e $(67,2 \%)$ dos agentes opinou que deve ser mantida a periodicidade do informativo impresso, ou seja, continuar a ser publicado semanalmente (Tabela 5).

\begin{tabular}{lcc}
\hline Periodicidade & Docentes & Agentes \\
\hline Semanal & $55,2 \%$ & $67,2 \%$ \\
Quinzenal & $38,9 \%$ & $20,0 \%$ \\
Mensal & $5,9 \%$ & $12,8 \%$ \\
\hline
\end{tabular}

Tabela 5: Opinião sobre a periodicidade da produção do Informativo UEM impresso.

Fonte: Elaborado pelo autor

A Tabela 6 mostra que $15 \%$ dos docentes e $20 \%$ dos agentes leem o informativo impresso semanalmente e são os mesmos que deram a opinião que o informativo deve continuar circulando semanalmente. $\mathrm{O}$ teste exato de Fisher mostrou haver associação entre a periodicidade de leitura e a opinião sobre a periodicidade da produção do Informativo impresso, para as duas categorias o p-valor foi menor que 0,05 . 


\begin{tabular}{l|rrr|rrr}
\hline \multirow{2}{*}{ Periodicidade } & \multicolumn{3}{|c|}{ Docentes } & \multicolumn{3}{c}{ Agentes } \\
\cline { 2 - 7 } & Semanal & Quinz. & Mensal & Semanal & Quinz. & Mensal \\
\hline Semanalmente & $15,0 \%$ & $6,3 \%$ & $0,4 \%$ & $20,0 \%$ & $3,4 \%$ & $1,3 \%$ \\
\hline Algumas & & & & & & \\
edições por mês & $8,0 \%$ & $7,2 \%$ & $0,8 \%$ & $7,4 \%$ & $4,6 \%$ & $1,9 \%$ \\
\hline Eventualmente & $4,9 \%$ & $6,1 \%$ & $1,7 \%$ & $5,9 \%$ & $1,9 \%$ & $3,2 \%$ \\
\hline
\end{tabular}

Tabela 6: Periodicidade com que leem versus opinião sobre periodicidade.

Fonte: Elaborado pelo autor

A tabela 7 refere-se à opinião dos leitores sobre o espaço reservado para publicação de artigos de opinião versus a intenção de escrever artigos de opinião. Nota-se que a maioria dos docentes, 36,1\% acha importante e sempre lê e gostariam de escrever artigos de opinião, já, entre os agentes, 43,7\% acha importante, sempre lê mas não tem interesse em escrever artigos. Verifica-se ainda que aproximadamente a metade dos docentes $(49,2 \%)$ gostaria, enquanto que a maioria $(82,1 \%)$ dos agentes não gostaria de escrever artigos de opinião.

O teste exato de Fisher mostrou haver associação (p-valor<0,05) das variáveis opinião sobre o espaço reservado para a publicação de artigos de opinião versus o interesse em escrever esse tipo de artigo, para as duas categorias.

\begin{tabular}{l|cc|cc}
\hline \multirow{2}{*}{ Opinião } & \multicolumn{2}{c|}{ Docentes } & \multicolumn{2}{c}{ Agentes } \\
\cline { 2 - 5 } & Sim & Não & Sim & Não \\
\hline Acho importante, sempre leio & $36,1 \%$ & $20,2 \%$ & $11,4 \%$ & $43,7 \%$ \\
Acho importante, mas não leio & $11,1 \%$ & $21,4 \%$ & $1,1 \%$ & $19,4 \%$ \\
Não deveria haver esse espaço & $0,0 \%$ & $2,8 \%$ & $0,4 \%$ & $5,7 \%$ \\
Não sabia da existência desse espaço & $2,0 \%$ & $6,3 \%$ & $4,9 \%$ & $13,3 \%$ \\
\hline
\end{tabular}

Tabela 7: Opinião sobre o espaço reservado para publicação de artigos de opinião versus interesse em escrever artigos de opinião

Fonte: Elaborado pelo autor

A Tabela 8 mostra a opinião dos entrevistados quanto ao conteúdo e o visual do Informativo UEM. A maioria dos docentes e agentes considera tanto o conteúdo quanto o visual do Informativo impresso como bom. No entanto, com relação ao visual um percentual considerável $30,8 \%$ de docentes e $17,5 \%$ dos agentes consideram regular ou ruim.

\begin{tabular}{l|l|l}
\hline Opinião & Conteúdo & Visual \\
\cline { 2 - 3 }
\end{tabular}




\begin{tabular}{l|cc|cc}
\cline { 2 - 4 } & Docentes & Agentes & Docentes & Agentes \\
\hline Ótimo & $17,0 \%$ & $17,5 \%$ & $2,4 \%$ & $14,1 \%$ \\
Bom & $69,6 \%$ & $72,2 \%$ & $62,1 \%$ & $61,2 \%$ \\
Indiferente & $3,6 \%$ & $2,3 \%$ & $4,7 \%$ & $7,2 \%$ \\
Regular & $8,7 \%$ & $7,2 \%$ & $22,9 \%$ & $14,8 \%$ \\
Ruim & $1,2 \%$ & $0,8 \%$ & $7,9 \%$ & $2,7 \%$
\end{tabular}

Tabela 8: Opinião dos entrevistados quanto ao conteúdo e o visual do Informativo UEM Fonte: Elaborado pelo autor

O principal motivo de interesse em ler o Informativo UEM é a busca por informações/soluções para suas necessidades, tanto para os docentes quanto para os agentes universitários. Outro interesse apontado pelos leitores é obter informações sobre as ações da reitoria (Tabela 9).

\begin{tabular}{lcc}
\hline \multicolumn{1}{c}{ Motivo } & Docentes & Agentes \\
\hline Para obter informação sobre as ações da Reitoria & $27,7 \%$ & $24,7 \%$ \\
Para encontrar informações/soluções para minhas necessidades & $32,8 \%$ & $44,8 \%$ \\
Para se informar sobre o que seus pares estão fazendo & $17,8 \%$ & $8,5 \%$ \\
Para se informar sobre a carreira & $9,5 \%$ & $13,9 \%$ \\
Para se informar sobre programas e convênios da universidade & $12,3 \%$ & $8,1 \%$ \\
\hline
\end{tabular}

Tabela 9: Motivo de interesse em ler o Informativo. Fonte: Elaborado pelo autor

\section{CONSIDERAÇÕES FINAIS}

O resultado mostrou que uma grande parte da comunidade universitária tem consciência sobre a importância do Informativo UEM. A maioria da comunidade universitária, docentes e agentes, leem o Informativo sendo que o impresso é o meio mais utilizado.

Ambas as categorias tem a opinião de que o informativo não deve deixar de circular na forma impressa e vir a ser substituído pela versão on-line.

Os principais motivos de interesse da comunidade universitária em ler o Informativo UEM é a busca por informações/soluções para suas necessidades e sobre as ações da Reitoria. 
Observou-se ainda que a maioria dos docentes e agentes considerou tanto o conteúdo quanto o visual do Informativo impresso como bom. No entanto, verificou-se pelos resultados, que um percentual considerável tem opinião de que deve haver melhorias no visual.

\section{REFERÊNCIAS}

BARBETTA, Pedro Alberto. Estatística Aplicada a Ciências Sociais. $6^{\text {a }}$ edição. Florianópolis: Ed. UFSC, 2006.

BARREIROS, T. E.; PAULA, C. de. A utilidade do jornal para o leitor: pesquisa com leitores da Gazeta do Povo (Curitiba-PR). In: $5^{\circ}$ ENCONTRO NACIONAL DE PESQUISADORES EM JORNALISMO. Universidade Federal de Sergipe - 15 a 17 de novembro de 2007.

BUSSAB, W. O.; MORETTIN, P. A. Estatística Básica. 5a edição. São Paulo: Ed. Saraiva, 2006.

CAMPOS, H. Estatística Experimental Não-Paramétrica, ESALQ, 1983.

PARIZOTTO, T. Informativo UEM conta a história da Universidade. Jornal da UEM, Paraná, nº 91, junho 2010.

RIGHETTI, S.; CARVALHO, R. Q.. Crise do jornalismo impresso e perspectivas para o futuro: um estudo dos dois maiores jornais diários impressos do Brasil. Intercom - Sociedade Brasileira de Estudos Interdisciplinares da Comunicação. XXXI Congresso Brasileiro de Ciências da Comunicação. Natal, RN, 2 a 6 de setembro de 2008.

SANTAELLA, Lúcia; Cultura das Mídias. 2a edição. São Paulo: Experimento, 2000.

O ESTAdO DE SÃo PAULO: Economia \& Negócios. Disponível em: <http://www.estadao.com.br/noticias/impresso,pesquisa-mostra-que-461-da-populacao-lejornal,568419,0.html>. Acesso em: 08 fev. 2012.

XAVIER, Paolo Philippe de Araújo. Problemas e perspectivas do jornal impresso. Observatório da Imprensa. ISSN 1519-7670 - Ano 17 - $\mathrm{n}^{\mathbf{0}}$ 723. Disponível em: $<$ http://www.observatoriodaimprensa.com.br/news/view/problemas_e_perspectivas_do_jornal impresso >. Acesso em: 8 fev. 2012. 